\title{
Téoros
}

Revue de recherche en tourisme

\section{Une éthique de la solidarité et de la responsabilité}

\section{Louis Jolin}

Volume 26, numéro 3, automne 2007

Tourisme et solidarité

URI : https://id.erudit.org/iderudit/1071000ar

DOI : https://doi.org/10.7202/1071000ar

Aller au sommaire du numéro

Éditeur(s)

Université du Québec à Montréal

ISSN

0712-8657 (imprimé)

1923-2705 (numérique)

Découvrir la revue

Citer ce document

Jolin, L. (2007). Une éthique de la solidarité et de la responsabilité. Téoros, 26(3), 3-5. https://doi.org/10.7202/1071000ar d'utilisation que vous pouvez consulter en ligne.

https://apropos.erudit.org/fr/usagers/politique-dutilisation/ 


\section{Une éthique de la solidarité et de la responsabilité}

\section{Louis Jolin}

Concept juridique ancien, la solidarité, lorsqu'elle est mentionnée explicitement, signifie en droit civil que les débiteurs d'un créancier sont obligés à une même chose et que chacun peut être contraint pour la totalité. En ce sens, la solidarité lie la responsabilité de chacun à celle de tous.

Terme à la mode, dont le sens peut varier selon le contexte d'application qui en privilégiera la dimension juridique, morale, philosophique, politique ou sociologique, la solidarité se veut une réponse aux nombreuses fractures sociales. "Exigence morale ou obligation publique ?» (Châtel, 2004), la solidarité se confond très souvent avec les concepts de charité, de justice, de fraternité, du devoir d'assistance...

Les écrits sur la solidarité ne datent pas d'hier - Léon Bourgeois a écrit son Essai philosophique de la solidarité en 1902 !-, mais ils se sont multipliés ces dernières années, peut-être parce que l'État-providence a perdu la faveur des discours politiques devant la montée du néo-libéralisme et de la mondialisation des marchés et qu'il devenait important d'apporter une réponse rassembleuse pour contrer leurs effets négatifs. La solidarité a investi alors le champ économique: ne parle-t-on pas de plus en plus d'économie solidaire, qui, accolée à "sociale", se présente comme une troisième voie entre l'économie publique et l'économique capitaliste?

Comment définir simplement la solidarité malgré ces diverses formes et les nombreux lieux où elle peut s'appliquer? Selon Jacques Milhau (1993: 75-77), on peut appréhender le concept de solidarité, même s'il recoupe "des solidarités de faits per-

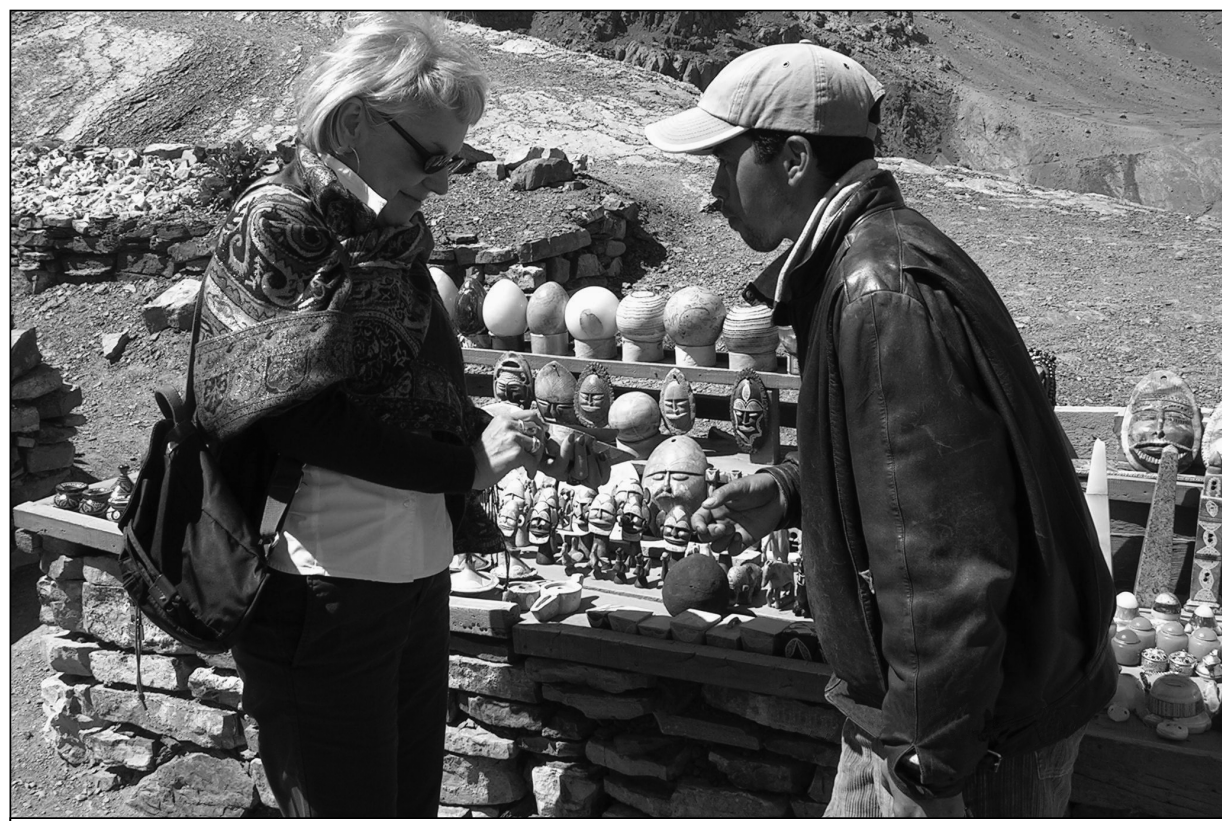

Atlas marocain, une transaction à juste prix?

Photo: Louis Jolin

ceptibles dans les domaines les plus divers ", allant jusqu'aux domaines biologique et mécanique, à partir d'une même catégorie de pensée qui le définit comme "une interdépendance d'interaction", comme une « réciprocité essentielle à la nature de ce qui est ainsi relié ou saisi comme tel à l'état syncrétique ».

Appliquée à la vie humaine, la solidarité postule que tous les hommes et les femmes de la Terre sont reliés entre eux et que, pour renforcer leur propre humanité, ils se doivent une aide mutuelle! Pour Raymond Chappuis (1999: 6), le concept de solidarité renvoie à la notion de solidité, "car rien n'est plus solide que le lien interhumain qui fait partie de l'équipement génétique de l'être de l'homme " et à la notion d'éthique qui «incite chacun à être responsable de lui-même et des autres ».
Déjà le concept juridique de solidarité renvoyait à celui de responsabilité. Ne disonsnous pas dans le jargon juridique que les débiteurs peuvent être "conjointement et solidairement responsables " ? Si la solidarité est une valeur suprême, au cœur d'une éthique de vie ou de relations humaines, elle conduit à la "responsabilité-pour-autrui » (Châtel, 2004). La responsabilité-pour-autrui est une solidarité d'appartenance. «L'appartenance à l'humanité contraint à la solidarité, solidarité qui est co-responsabilité face à toute injustice, face à tout acte commis et notamment quand ces actes sont connus " (Idem, 2004 : 160).

C'est ainsi que l'éthique de la solidarité rejoint celle de la responsabilité. Le questionnement éthique sur les conséquences pour autrui fait émerger non seulement la responsabilité des individus les uns par 
rapport aux autres, mais également la responsabilité sociale des entreprises, c'est-à-dire l'obligation qu'elles ont de répondre de leurs actions envers l'ensemble de leurs parties prenantes et plus spécialement envers les collectivités humaines dans lesquelles elles interviennent.

Mais comment exercer concrètement la solidarité ou la responsabilité-pour-autrui dans un monde dominé par l'argent et par les forces du marché? Avant tout, il s'agit de la voir comme une force d'interpellation qui nous oblige à nous questionner sur nos comportements et à tendre par divers moyens vers l'Autre. Au-delà du simple respect de codes et de chartes, fort utiles par ailleurs, il y a l'éthique de la responsabilisation qui « propose de mettre en cause et en question ce qui est au profit de l'essai et de la proposition, en prenant en charge les conséquences de l'action à partir de délibérations et d'une solidarité qui devrait constituer le fond de l'existence collective » (Desbons et Ruby, 2004 : 48).

\section{Le tourisme, un terrain propice pour la solidarité, la responsabilité et la responsabilisation}

Le tourisme n'est pas qu'un terme générique qui recouvre un ensemble d'activités déployées par des personnes au cours de leurs voyages. Pour plusieurs auteurs, le sens profond de l'activité touristique réside dans la découverte et la rencontre d'un ailleurs plus ou moins éloigné, constitué de lieux et de personnes. Le tourisme constitue un "apprentissage de l'altérité " (Delisle et Jolin, $2007: 7$ ).

Malheureusement, même si le tourisme est un réel facteur de développement social, culturel et économique, mal maîtrisé et laissé aux seules mains des promoteurs et des développeurs de toutes sortes, il peut avoir plus d'effets négatifs que positifs. La prise de conscience, ces dernières années, des effets équivoques du tourisme a induit une réflexion critique et éthique sur l'exercice de l'activité touristique. Comment faire pour que le tourisme devienne réellement une activité bénéfique pour les visiteurs comme pour les visités et qu'il soit réellement une activité de découverte et de rencontre de l'Autre?

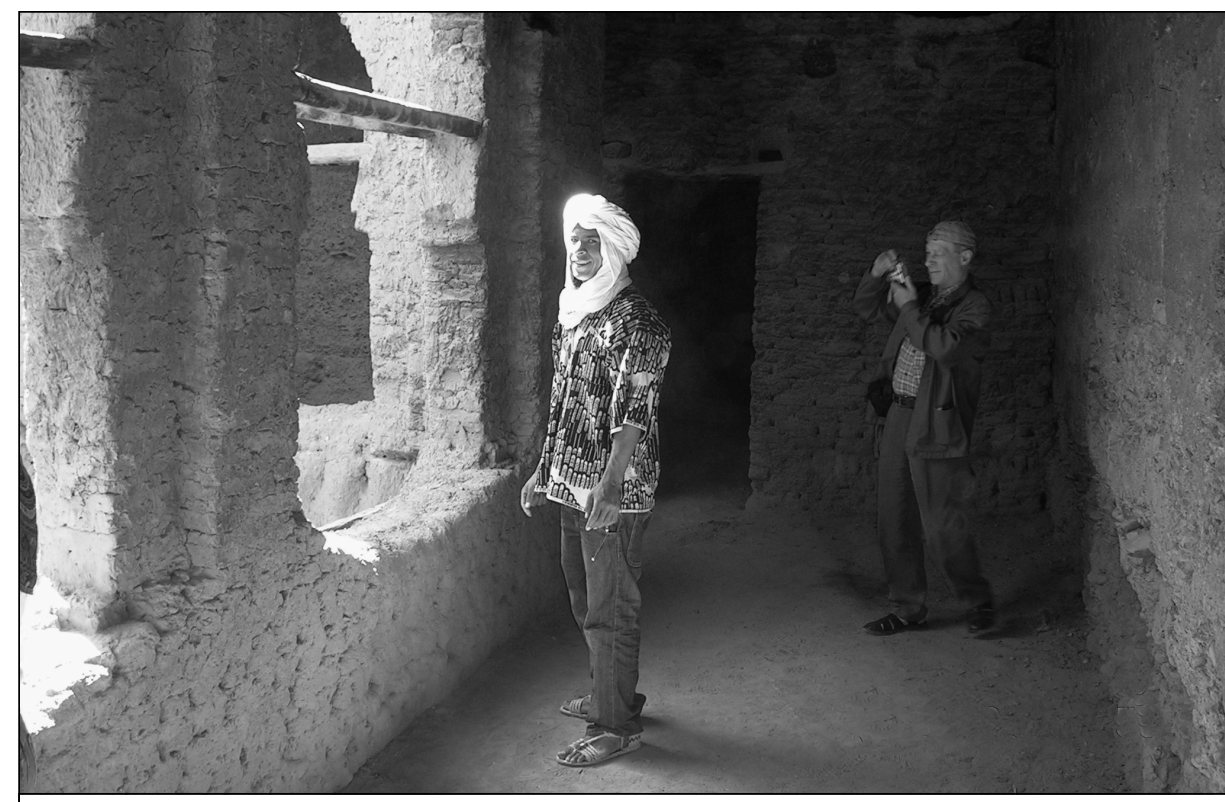

Guide et tourisme, un double regard, Maroc.

Photo: Louis Jolin

Cette question a donné lieu à la production de chartes et de codes éthiques - dont le plus connu est certainement le Code mondial d'éthique du tourisme de l'Organisation mondiale du tourisme - pour guider les acteurs de la chaîne touristique dans leurs divers comportements, à commencer par les touristes eux-mêmes. Elle a surtout donné lieu à diverses propositions, mises de l'avant par des communautés locales, des associations, des ONG (organisations non gouvernementales) de coopération, des voyagistes, des chercheurs, dans le sens d'une plus grande responsabilisation et d'une recherche de solidarité. Car le tourisme, comme lieu d'apprentissage de l'altérité, recèle un fort potentiel pour exprimer notre solidarité et notre appartenance à l'humanité. II peut être l'occasion d'exprimer une solidarité envers les personnes qui ont divers types de contraintes en raison de leur situation financière ou d'une incapacité quelconque, envers les autochtones des territoires que l'on souhaite visiter, envers les pays en développement et leurs populations, envers les régions en difficulté des pays industrialisés.

Les moyens d'expression de la solidarité en tourisme sont multiples et passent par une rémunération équitable des travailleurs et des producteurs locaux, par une participation des populations à la définition de leurs projets, par un appui concret de la part des visiteurs à des projets de développement dans les pays visités, par des mesures de protection de l'environnement et du patrimoine naturel et culturel des lieux visités, par des programmes de subventions, à la pierre et à la personne, afin d'aider les plus démunis à participer à l'activité touristique...

Un « autre tourisme » a ainsi vu le jour, qui s'exprime par diverses expressions qui, bien que reposant sur un socle de valeurs communes, visent à apporter diverses nuances. Tourisme responsable, tourisme social, tourisme solidaire, tourisme intégré, tourisme équitable, tourisme communautaire sont quelques-unes de ces expressions qui réfèrent directement à des valeurs, dont celles de la responsabilité et de la solidarité. D'autres expressions comme l'écotourisme, le tourisme culturel ou encore le congé solidaire réfèrent davantage à des formes et à des pratiques qui prennent de plus en plus de place dans l'offre touristique mondiale, permettant d'exprimer, bien que pas toujours, des niveaux de conscientisation, de responsabilisation et de solidarité (Delisle et Jolin, 2004 : 39-76).

Le présent dossier thématique n'a pas la prétention d'explorer toutes les formes de solidarité que peut recéler l'activité touristique. II met principalement l'accent sur le tourisme dit solidaire, avec quelques incursions sur le tourisme social, le tourisme communautaire, le congé solidaire et le tourisme en milieu autochtone. 
Le tourisme solidaire « regroupe les formes de tourisme alternatif qui mettent au centre du voyage l'homme et la rencontre, et qui s'inscrivent dans une logique de développement des territoires » (UNAT, 2002 : 4). La solidarité s'exprime, entre autres, par l'appui ou la participation des voyageurs et des voyagistes, y compris des associations et des ONG de coopération à un projet de développement dont la pérennité est prévue grâce à des actions qui s'inscrivent dans la durée.

Le tourisme solidaire se fonde sur une critique du tourisme de masse pour promouvoir un partage plus juste des retombées du tourisme, de telle sorte que les populations des communautés d'accueil puissent réellement en bénéficier. Au début de notre dossier thématique, Isabelle Sacareau présente les acteurs de ce tourisme et leurs modalités d'action, tout en analysant de façon critique ses ressorts, tant du point de vue de ses promoteurs que des touristes. Elle s'interroge aussi sur la capacité du tourisme solidaire d'initier un véritable processus de développement par rapport aux autres formes de tourisme.

Bernard Schéou propose une réflexion sur le temps du tourisme. Le temps des vacances semble soumis, dans les faits, aux mêmes contraintes que celles qui s'appliquent aux activités du quotidien. Mais comment rejoindre l'Autre si le tourisme de masse est conçu, sur les plans spatial et temporel, pour empêcher les gens de se rencontrer? L'auteur se demande si la lenteur ne devient pas alors une condition essentielle pour un véritable tourisme équitable et solidaire. Le « tourisme lent » s'inscrit dans la même mouvance que ce que défend une organisation internationale comme Slow Food dans le secteur de la restauration. Pour Schéou, il faut trouver le bon rythme, une sorte d'eurythmie, qui, en s'adaptant au rythme des populations visitées, favorise la rencontre et l'échange.

Même si les arrivées des touristes internationaux et les recettes du tourisme international sont en progression en Afrique, la part de ce marché à l'échelle mondiale reste encore faible. Le tourisme social et le tourisme solidaire peuvent-ils représenter des voies appropriées pour développer le potentiel touristique de l'Afrique en complémentarité au tourisme international traditionnel ? Pour répondre à cette question, le Bureau international du tourisme social (BITS) a réalisé une importante étude avec la col- laboration de l'Organisation mondiale du tourisme (OMT), du ministère français des Affaires étrangères et de l'Union nationale des associations de tourisme (UNAT-France). Charles Bélanger, Louis Jolin et Jean-Marc Mignon, coordonnateurs de l'étude, rendent compte des principaux faits saillants de ce travail, qui comprend une réflexion sur les concepts du tourisme social et solidaire dans une perspective d'application dans le continent africain, une enquête auprès des administrations nationales du tourisme en Afrique et une enquête auprès des acteurs locaux du tourisme solidaire de ce continent.

Dans le prolongement de cette recherche, Mimoun Hillali propose une critique des aides spéculatives du passé consenties à l'Afrique, tout en soulignant les aspects positifs des solidarités mécaniques. L'auteur propose une réflexion sur le lien entre solidarité et sociabilité dans l'acte touristique bien planifié et il s'interroge sur la version mi-moderrne et mi-traditionnelle de l'entraide coutumière africaine prônée par les adeptes du tourisme solidaire.

Sylvie Blangy et Alain Laurent, s'appuyant sur de l'information qui provient d'études de cas de divers coins de la planète, présentent le tourisme autochtone (ou indigène) comme un lieu privilégié pour des formes de solidarité innovantes : au sein des communautés ellesmêmes, mais aussi entre les communautés et les exploitants touristiques, entre les communautés et les voyageurs, entre les communautés et les scientifiques... Les communautés autochtones, par le biais de leurs expériences d'accueil touristique, malgré les difficultés, révèlent des formes d'organisation et de gestion qui peuvent être une source d'inspiration pour nos sociétés occidentales.

La formule du congé solidaire, popularisée en France par l'association Planète Urgence, est maintenant disponible au Canada grâce à une entente entre cette association et deux organismes de coopération internationale : le Centre d'étude et de coopération internationale (CECI), basé à Montréal, et l'Entraide universitaire mondiale du Canada, dont le siège est à Ottawa. Melina Schoenborn présente cette formule qui permet d'effectuer un travail humanitaire lié à la profession du participant pendant une partie de ses vacances annuelles ou lors d'un congé sans traitement. Mais s'agit-il d'une forme de tourisme ou d'une nouvelle forme de coopération internationale?
Le tourisme solidaire -il en est de même du tourisme communautaire - est surtout vécu dans un axe Nord-Sud. Mais peut-il se vivre au sein des pays industrialisés du Nord ? C'est à cette question que répond Marie-Andrée Delisle en analysant une expérience de tourisme communautaire dans une collectivité des Hautes-Laurentides, au Québec. II s'agit d'un village d'accueil "nouvelle génération » qui propose une expérience humaine significative pour le visiteur et le visité et qui implique une forte dose de solidarité entre les villageois.

La solidarité n'est pas qu'un simple mot. Sa mise en œuvre en tourisme, comme le révèlent les divers articles du dossier, en est la preuve. Les formes de solidarité en tourisme sont multiples bien que non achevées. Force d'interpellation, la solidarité comme concept peut remettre en question nos façons de faire et nous obliger à changer notre mentalité... La solidarité vécue dans nos activités quotidiennes comme en périodes de vacances, lors de voyages touristiques, se construit au jour le jour et demeure exigeante, mais n'est-elle pas une condition d'« une vie authentiquement humaine sur terre » (Jonas)?

Louis Jolin est professeur au Département d'études urbaines et touristiques de l'École des sciences de la gestion de l'Université du Québec à Montréal.

\section{Bibliographie}

Bourgeois, Léon 1902 [1998], Solidarité, Villeneuve d'Ascq, Presses universitaires du Septentrion.

Chappuis, Raymond (1999), La solidarité. L'éthique des relations humaines, Paris, Presses universitaires de France.

Châtel, Vivianne (2004), "La solidarité : une exigence de 'responsabilité-pour-autrui'? ", dans Marc-Henry Soulet (dir.), La solidarité : exigence morale ou obligation publique?, Fribourg, Academic Press Fribourg, p. 138-162.

Delisle, Marie-Andrée, et Louis Jolin (2007), Un autre tourisme est-il possible?, Québec, Presses de l'Université du Québec.

Desbons, David, et Christian Ruby (2004), La responsabilité, Paris, Quintette.

Milhau, Jacques (1993), Solidarité : l'avenir d'un héritage, Paris, Éditions sociales.

UNAT (2002), D'autres voyages, du tourisme à l'échange, Paris, Union nationale des associations de tourisme. 